\title{
Anomalous muscle from the fascia around popliteal vessels
}

\author{
Rodrigues $\mathrm{V}^{1}$, Satheesha Nayak B ${ }^{2}$, Mohandas Rao $\mathrm{KG}^{2}$, Rao $\mathrm{AS}^{3}$, Venkataramana $\mathrm{V}^{2}$, \\ Somayaji $\mathrm{SN}^{2}$
}

\author{
Department of Anatomy, Faculty of Medicine, University of West Indies, Trinidad \\ mohandaskg@gmail.com
}

\begin{abstract}
Normally, the popliteal fossa contains popliteal vessels, tibial and common peroneal nerves. An occurrence of muscle in the popliteal fossa is very rare. During routine dissection classes for medical undergraduates, an anomalous muscle in the popliteal fossa was encountered. The muscle was originating from the thick fascia around the popliteal vessels. It was getting inserted to the medial head of the gastrocnemius through a narrow tendon and was supplied by a branch of tibial nerve. The muscle was observed in the right limb and was unilateral. An awareness of the possibility that such anomalous muscles can occur in the popliteal fossa is clinically essential as these may possibly entrap and compress the popliteal vessels. The muscle reported in the present case bears clinical significance since near its origin it almost completely surrounded the popliteal vessels and could pull on these vessels on contraction (Fig. 3, Ref. 14). Full Text in PDF www.elis.sk.

Key words: popliteal fossa, abnormal muscle, anomalous muscle, popliteal vessels, compression, entrapment.
\end{abstract}

Popliteal fossa is a diamond-shaped space situated behind the knee joint. Its superolateral, superomedial, inferolateral, and inferomedial boundaries are formed by biceps femoris, semitendinosus and semimembranosus muscles, lateral head of gastrocnemius muscle, and medial head of the gastrocnemius muscle, respectively. The roof is formed by the popliteal fascia whereas the floor is formed by the popliteal surface of the femur, capsule of the knee joint with oblique popliteal ligament, and the fascia covering the popliteus muscle. The fossa contains popliteal vessels and tibial and common peroneal nerves as well as their branches. Normally the popliteal fossa does not contain any muscle (1). However; there are few reports of muscles occurring in the popliteal fossa. The muscle reported in the present case is very unique as it takes its origin from the fascia surrounding the popliteal vessels.

\section{Case presentation}

During routine dissection for undergraduate medical students, we observed an anomalous muscle in the popliteal fossa of an elderly male Indian cadaver. The anomaly was in the right limb and it was unilateral. The abnormal muscle took its origin from the thick fascia covering the popliteal vessels (Figs 1, 2 and 3). Its fleshy fibers surrounded the popliteal vessels as well as the nerve to the lateral head of the gastrocnemius. The muscle reduced into a narrow tendon which passed downward and medially, crossed

${ }^{1}$ Department of Anatomy, Faculty of Medicine, University of West Indies, Trinidad, ${ }^{2}$ Department of Anatomy, Melaka Manipal Medical College, Manipal, University Manipal, India, and ${ }^{3}$ Department of Orthopedics, Faculty of Medicine, AIMST University, Semeling, Bedong, Kedah, Malaysia

Address for correspondence: Mohandas Rao KG, MD, Melaka Manipal Medical College, Manipal University, 576104 Manipal, India.

Phone: +910844380839 superficially to the sural nerve and got inserted into the medial head of the gastrocnemius muscle. The muscle derived its nerve supply from a branch of tibial nerve.

\section{Discussion}

It is not common for anomalous muscles to be present in the popliteal fossa. Somayaji et al. have reported a muscle originating from the semimembranosus and biceps femoris muscles and then getting inserted into the superficial surface of the calcaneal tendon (2). An insertion of muscle slips from biceps femoris into gastrocnemius muscle and calcaneal tendon has also been reported by Moore (3). Parsons has reported a muscle slip passing transversely between the two heads of origin of the gastrocnemius (4). According to Barry and Bothroyd, extra slips of origin associated with gastrocnemius and soleus usually join those muscles or the calcaneal tendon (5). Jaijesh et al., have reported an abnormal muscle originating from two tendinous slips; one from the lateral intermuscular septum and the other from the posterior intermuscular septum of the thigh (6). These two slips then united and formed a muscular belly in front of the lower end of the sciatic nerve and then were inserted into both heads of the gastrocnemius muscle.

The most common anomalous muscle seen in the popliteal fossa is the third head (caput tertium) of gastrocnemius $(7,8)$. It may arise from the long head of biceps femoris or from more than one location, or it may divide near its termination to join both heads of gastrocnemius. The third head may or may not cross the popliteal neurovascular structures. It has an overall frequency of 2.9 to $5.5 \%$. Gupta et al have reported a "Y" shaped anomalous muscle in the popliteal fossa (9). The presence of aberrant popliteal muscle and its clinical importance have been discussed by Banjo (10). 


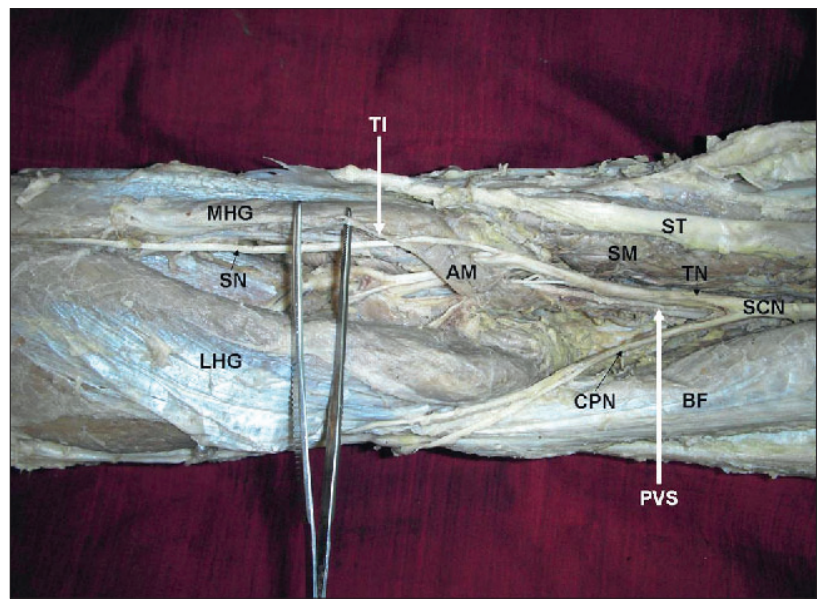

Fig. 1. Photograph of the popliteal fossa showing the anomalous muscle. The picture illustrates the popliteal fossa exposed from behind showing the anomalous muscle (AM) with its tendon of insertion (TI) getting inserted to the medial head of gastrocnemius (MHG). (SCN sciatic nerve; ST - semitendinosus; BF - biceps femoris; SM - semimembranosus; TN - tibial nerve; $\mathrm{CPN}$ - common peroneal nerve; LHG - lateral head of gastrocnemius; SN - sural nerve, PVS - popliteal vessels).

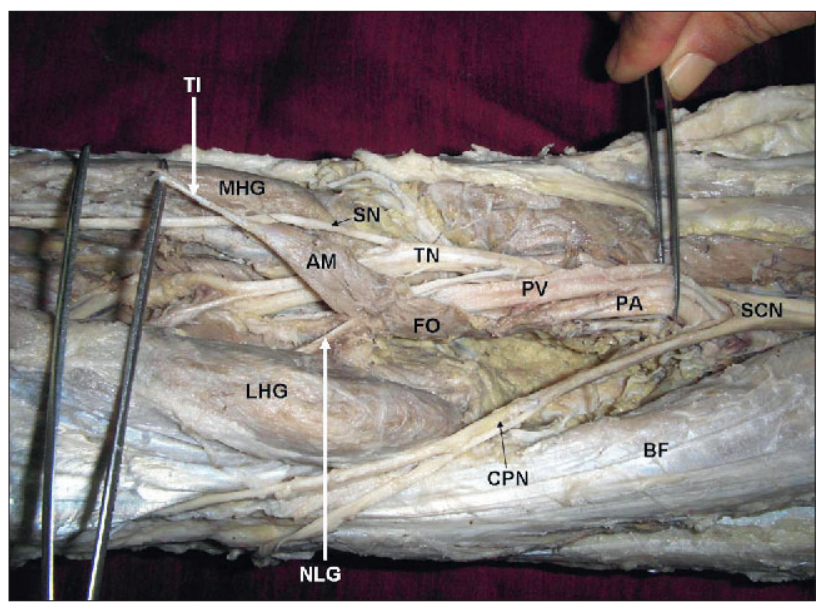

Fig. 2. Photograph of the closer view of the popliteal fossa showing the anomalous muscle. The picture illustrates the popliteal fossa exposed from behind showing the anomalous muscle (AM) taking its origin through fleshy fibers (FO) from the connective tissue around the popliteal artery (PA), popliteal vein (PV), and nerve to the lateral head of gastocnemius (NLG), and getting inserted through a tendon (TI) to the medial head of gastrocnemius (MHG). It can also be noted that the muscle is crossing over the sural nerve (SN) as it is passing towards its insertion. ( $\mathrm{SCN}$ - sciatic nerve; $\mathrm{BF}$ - biceps femoris; TN - tibial nerve; CPN - common peroneal nerve; LHG - lateral head of gastrocnemius).

To our knowledge, no case of anomalous muscle in the popliteal fossa taking its origin from the connective tissue sheath around the polpiteal vessels (as in the present case) has been reported.

Additional muscles and an abnormal attachment of muscles in the region of popliteal fossa may compress or entrap the sciatic, tibial or common peroneal nerve. They may also entrap and compress the popliteal vessels. A case of popliteal artery entrapped

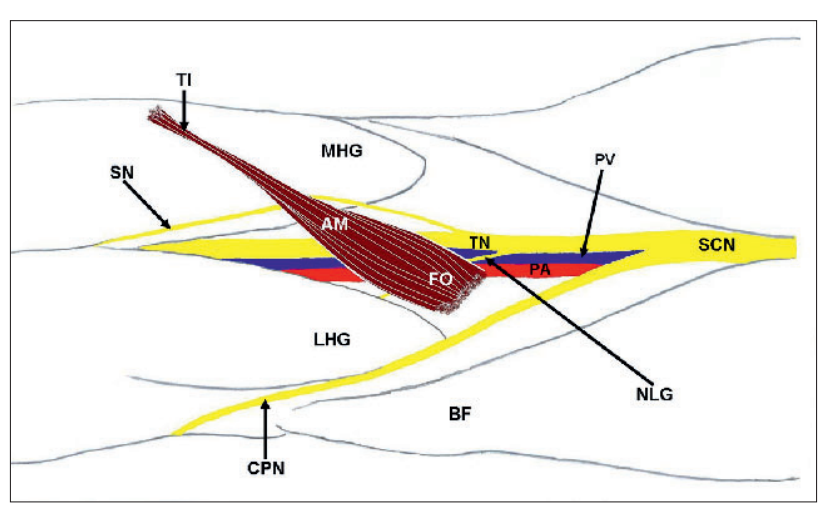

Fig. 3. Schematic picture of the popliteal fossa showing the anomalous muscle. The picture illustrates the popliteal fossa showing the anomalous muscle (AM) taking its origin through fleshy fibers (FO) from the connective tissue around the popliteal artery (PA), popliteal vein (PV) and nerve to the lateral head of gastocnemius (NLG), and getting inserted through a tendon (TI) to the medial head of gastrocnemius (MHG). It can also be noted that the muscle is crossing over the sural nerve (SN) as it is passing towards its insertion. ( $\mathrm{SCN}$ - sciatic nerve; $\mathrm{BF}$ - biceps femoris; TN - tibial nerve; CPN - common peroneal nerve; LHG - lateral head of gastrocnemius).

by an abnormal medial head of gastrocnemius muscle has been reported (11).

The presently reported case of additional muscle becomes clinically more significant than those of other additional muscles reported in this region because of its peculiar origin. As the fleshy fibers of this muscle are arising from the connective tissue around the popliteal vessels they may cause popliteal vessel entrapment and compression, especially in athletes and body builders, in whom such muscles might get hypertrophied. The muscle also entrapped the nerve to the lateral head of the gastrocnemius, and its tendon passed over the sural nerve which could result in compression of these nerves.

Despite the fact that this muscle represents no functional advantage, it is possible that its contraction can pull on the popliteal vessels and produce clinical symptoms of popliteal vessel entrapment syndrome.

Popliteal artery entrapment syndrome (PAES) is a rare but a potentially limb-threatening peripheral vascular problem occurring predominantly in young adults. The incidence of PAES is stated to be only $0.16 \%$ to $3.5 \%$ (12). In 1879, Anderson Stuart, an Edinburgh medical student, described an anatomical variant of the popliteal artery which he had dissected from a gangrenous limb. The significance of this anomaly was not recognized until 1959 when Hamming and Vink described the clinical syndrome which is associated with entrapment of the popliteal artery (12).

PAES is caused by an anomalous relationship of muscle and artery in the popliteal fossa resulting in extrinsic arterial compression. Popliteal vessel entrapment syndrome has been cited to occur due to various anomalies of the vessel or surrounding structures. Abnormal embryologic development can result in numerous anomalous popliteal fossa relationships responsible for the entrapment (13). The anomalies varied from abnormal courses of vessels, abnormal attachment of muscles and tendons, presence of abnormal 
fibrous bands and abnormal deep muscles in the popliteal region in the vicinity of the popliteal vessels. All these, by way of compression of the popliteal vessels, could present with clinical features of such compression requiring medical or surgical intervention (12). The chief complaints were progressive intermittent claudication and foot coldness during exercise with symptom duration ranging from eight months to two years (12).

The abnormal muscles described in the popliteal region can be bilateral. The muscles may be placed superficially or deep in the popliteal fossa. The superficially placed muscles may remain asymptomatic; the deeply placed abnormal muscles lying in the vicinity of vessels can become symptomatic. Some of these muscles can also be hypertrophied to produce these symptoms especially in athletic and bodybuilding individuals. They can produce severe vascular symptoms due to repetitive insults to the popliteal artery causing arterial damage, aneurysms, thromboembolism or arterial thrombosis requiring major surgical interventions (14). Once detected, popliteal artery entrapment syndrome should be treated by surgery despite the degree of symptoms. Because of the progressive nature of this entity, even asymptomatic limbs should have surgical correction to prevent irreversible change (13).

Hence, the knowledge of presence of such abnormal muscles producing clinical vascular symptoms is of paramount importance to the investigating radiologist and the surgeon. Duplex sonography, CT, MRI, and angiography have all been used in investigating such symptoms in patients. We would imagine that such nonessential muscles could also be considered for surgical procedures as muscle grafting.

\section{Conclusion}

To conclude, we would like to state that the muscle reported by us in this case is unique in its origin, and will supplement our knowledge of variations of muscles in this region. Not only for anatomists but also for radiologists and surgeons, it is very essential to be aware of the probable occurrence of additional muscles in this region for proper diagnosing and operative planning of treatment.

\section{References}

1. Standring S, Ellis H, Healy JC, Johnson D, Williams A, Collins P. Gray's Anatomy: The Anatomical Basis of Clinical Practice. London: Elsevier, Churchill Liwingstone, 2005: 1471.

2. Somayaji SN, Vincent R, Bairy KL. An anomalous muscle in the region of the popliteal fossa: case report. J Anat 1998; 192: 307-308.

3. Moore AT. An anomalous connection of the piriformis and biceps femoris muscles. Anat Rec 1922; 23: 307-314.

4. Parsons FG. Note on an abnormal muscle in the popliteal space. J Anat 1920; 54: 170-178.

5. Barry D, Bothroyd JS. Tensor fasciae suralis. J Anat 1924; 58: 382-390.

6. Jaijesh P, Venkata RV, Satheesha N. Sciatic nerve entrapment in the popliteal fossa: a case report. Neuroanatomy 2005; 4: 41-42.

7. Bergman RA, Walker CW, el-Khour GY. The third head of Gastrocnemius in CT images. Annals Anat 1995; 177: 291-294.

8. Williams PL, Bannister LH, Berry MM et al. Haemolymphoid system. 1423-1431. In: Gray's Anatomy. 38th Edition; ELBS with Churchill Livingstone, Edinburgh, London. 1995.

9. Gupta RK, Bhagwat SS. An anomalous muscle in the region of the popliteal fossa: A case report. J Anat Soc India 2006; 55: 65-68.

10. Banjo AO. Aberrant popliteus muscle: anatomy and clinical consideration. Afr J Med Med Sci 1996; 25: 69-73.

11. Tamaki Y, Sano A, Okada T et al. Bilateral popliteal artery entrapment syndrome: reemphasis on reading axial tomograms. Radiat Med 2007; 25: 548-552.

12. Gourgiotis S, Aggelakas J, Salemis N, Elias C, Georgiou C. Diagnosis and surgical approach of popliteal artery entrapment syndrome: a retrospective study. Vasc Health Risk Manag 2008; 4: 83-88.

13. Macedo TA, Johnson CM, Hallett JW Jr, Breen JF. Popliteal artery entrapment syndrome: role of imaging in the diagnosis. AJR Am J Roentgenol 2003, 181: 1259-1265.

14. de OliveiraI FM, SantosI ACB, TakitoII AM, BolanhoII E, da CostaIII, RFB, Fernandes N. Bilateral popliteal artery entrapment syndrome: case report. J Vasc Bras 2008; 7 (2). doi: 10.1590/S16774492008000200011. 